to target multiple antigens is required. We propose a controlled ACT approach, where T cells are armed with synthetic agonistic receptors (SARs) that are conditionally activated only in the presence of a target AML-associated antigen, and a cross-linking bispecific $\mathrm{T}$ cell engager (BiTE) specific for both (SAR) $\mathrm{T}$ cell and tumour cell.

Materials and Methods A SAR composed of an extracellular EGFRvIII, trans-membrane CD28, and intracellular CD28 and CD3z domains was fused via overlap-extension PCR cloning. $\mathrm{T}$ cells were retrovirally transduced to stably express our SAR construct. SAR-specific bispecific $\mathrm{T}$ cell engagers (BiTE) that target AML-associated antigens were designed and expressed in Expi293F $\mathrm{F}^{\mathrm{TM}}$ cells and purified by nickel affinity and size exclusion chromatography (SEC). We validated our approach in three human cancer models and patient-derived AML blasts expressing our AML-associated target antigen CD33.

Results CD33-EGFRvIII BiTE, monovalently selective for our SAR, induced conditional antigen-dependent activation, proliferation and differentiation of SAR-T cells. Further, SAR T cells bridged to their target cells by BiTE could form functional immunological synapses, resulting in efficient tumor cell lysis with specificity towards CD33-expressing AML cells. SAR.BiTE combination could also mediate specific cytotoxicity against patient-derived AML blasts whilst driving SAR T cell activation. In vivo, treatment with SAR.BiTE combination could efficiently eradicate leukemia and enhance survival in an AML xenograft model. Furthermore, we could show selective activation of SAR $\mathrm{T}$ cells, as well as a controllable reversibility of said activation upon depletion of the $\mathrm{T}$ cell engaging molecule.

Conclusions Here we apply the SAR $x$ BiAb approach in efforts to deliver specific and conditional activation of agonistic receptor-transduced $\mathrm{T}$ cells, and targeted tumour cell lysis. The modularity of our platform will allow for a multi-targeting ACT approach with the potential to translate the ACT successes of B cell malignancies to AML. With a lack of truly specific AML antigens, it is invaluable that this approach possesses an intrinsic safety switch via its BiTE facet. Moreover, we are able to circumvent pan-T cell activation due to the specific targeting and activation of SAR T cells.

Disclosure Information M. Benmebarek: None. B.L. Cadilha: None. M. Hermann: None. S. Lesch: None. C. Augsburger: None. B. Brauchle: None. S. Stoiber: None. A. Darwich: None. F. Rataj: None. C. Klein: A. Employment (full or parttime); Significant; Roche. K. Hopfner: None. M. Subklewe: None. S. Endres: None. S. Kobold: None.

\section{L5 RIG-I ACTIVATION ENHANCES MELANOMA IMMUNOGENICITY AND IMPROVES ANTI-TUMOR T CELL RESPONSES IN COMBINATION WITH ANTI-PD-1 IMMUNE CHECKPOINT BLOCKING ANTIBODIES}

\footnotetext{
1,2 $\mathrm{B}$ Thier* 1,2 L Such, 1,2 M Schwamborn, $1,2 \mathrm{~A}$ Sucker, ${ }^{3} \mathrm{C}$ Coch, $1,2 \mathrm{D}$ Schadendorf, ${ }^{1,2} \mathrm{~K}$ Griewank, ${ }^{4} \mathrm{M}$ Trilling, ${ }^{1,2} \mathrm{~F}$ Zhao, ${ }^{1,2} \mathrm{~A}$ Paschen. ${ }^{1}$ Dermatology, University Hospital Essen, Essen, Germany; ${ }^{2}$ German Cancer Consortium (DKTK), Partner Site Essen/Düsseldorf, Essen, Germany; ${ }^{3}$ Institute of Clinical Chemistry and Clinical Pharmacology, University Hospital Bonn, Bonn, Germany; ${ }^{4}$ Institute of Virology, University Hospital Essen, Essen, Germany
}

\subsection{6/jitc-2020-ITOC7.5}

Background Clinical efficacy of immune checkpoint blocking (ICB) therapy critically relies on the killing of melanoma cells by $\mathrm{CD}^{+} \mathrm{T}$ cells, becoming activated upon recognition of tumor antigens presented by HLA class I (HLA-I) surface molecules. Patient-derived melanoma cells can escape from cytotoxic $\mathrm{T}$ cell effector functions by loss of HLA-I surface expression due to the silencing of HLA-I antigen processing and presentation machinery (APM) genes.

Material and Methods Seeking for a strategy to restore HLA-I expression, we transfected melanoma cells obtained from distinct patient metastasis with synthetic short double stranded RNA (3pRNA), an activating ligand of the cytosolic innate pattern recognition receptor RIG-I. 3pRNA-transfected melanoma cells were analyzed for HLA-I surface expression by FACS analysis and gene expression of HLA-I APM components by qPCR. In vivo 3pRNA-transfected tumors were analyzed for HLA-I expression by immunohistochemistry staining. Furthermore, $T$ cell activation after coincubation with 3 pRNAtransfected melanoma cells was determined by IFN $\gamma$-ELISpot assay. The effect of combined 3pRNA and blocking anti-PD-1 antibody treatment on $\mathrm{T}$ cell activation was measured by intracellular cytokine staining and FACS analysis.

Results Activation of RIG-I by 3pRNA increased the expression of HLA-I APM components and strongly enhanced recognition of melanoma cells by autologous $\mathrm{CD} 8^{+} \mathrm{T}$ cells. Based on these findings, we asked whether the combination of 3 pRNA and blocking anti-PD-1 antibodies could improve antimelanoma $\mathrm{T}$ cell responses in an anti-PD-1 non-responder patient model. Indeed, $\mathrm{T}$ cell activation by 3 pRNA-transfected melanoma cells was significantly increased in the presence of anti-PD-1 antibodies. In line with the enhancement of antitumor $\mathrm{T}$ cell responses, we found an association of elevated RIG-I mRNA levels with prolonged patient survival in TCGA melanoma samples.

Conclusions In summary, this study demonstrates a beneficial effect of RIG-I activation on antigen presentation and $\mathrm{T}$ cell recognition of melanoma cells. Improved $\mathrm{T}$ cell responses by combined 3pRNA and anti-PD-1 treatment suggests that combinational therapy could be a strategy to overcome $\mathrm{T}$ cell resistance in melanoma.

Disclosure Information B. Thier: None. L. Such: None. M. Schwamborn: None. A. Sucker: None. C. Coch: None. D. Schadendorf: None. K. Griewank: None. M. Trilling: None. F. Zhao: None. A. Paschen: None.

\section{On Demand Talks}

\section{On Demand Talks: Tumor Microenvironment}

\section{TUMOR LACTIC ACIDOSIS ALTERS DECISIVE T CELL ACTIVITIES}

${ }^{1}$ AJ Fischbeck*, ${ }^{1}$ AN Mendler, ${ }^{2} M$ Balles, ${ }^{2}$ J Schwarz, ${ }^{2} \mathrm{R}$ Zantl, ${ }^{1} E$ Noessner. ${ }^{1}$ Helmholtz Zentrum München, Immunoanalytics, Munich, Germany; ${ }^{2} I B I D I$ GmbH, Gräfelfing, Germany

\subsection{6/jitc-2020-ITOC7.6}

Background Adoptive $\mathrm{T}$ cell therapy is a promising treatment strategy for tumor patients. However, when entering the tumor microenvironment (TME), $\mathrm{T}$ cells lose their effector function showing reduced degranulation and cytokine secretion. Besides $\mathrm{T}$ cell inhibition through checkpoint pathways (i. e. PD-1/L1, CTLA-4), suppressor cells (i.e. TAM, $\mathrm{T}_{\text {reg }}$ ) and cytokines (i.e. IL-10, TGF, VEGF), various metabolites of the TME also counteract antitumoral activities. Among the latter, lactate and extracellular acidosis are byproducts of the cancer metabolism and commonly observed in high concentrations in 